\title{
Adaptive Tuning of MIMO-enabled 802.11e WLANs with Network Utility Maximization
}

\author{
Yuxia Lin and Vincent W.S. Wong \\ Department of Electrical and Computer Engineering \\ The University of British Columbia, Vancouver, BC, Canada, V6T 1 Z4 \\ Email: \{yuxial, vincentw\} @ece.ubc.ca
}

\begin{abstract}
The IEEE 802.11-based wireless local area networks (WLANs) are widely used for high-speed wireless data access. With the recent 802.11e quality-of-service $(\mathrm{QoS})$ extension, realtime applications such as voice over IP and video streaming are finding their way to be running over WLANs. The recent 802.11n proposal aims to provide higher throughput support for bandwidth-intensive multimedia applications. It uses the multiple-input-multiple-output (MIMO) technology at the physical layer to increase the transmission rate. MIMO introduces several new features at the physical layer such as the spatial diversity and spatial multiplexing gains. These new characteristics at the wireless physical layer require corresponding adaptation at higher layers to achieve a better performance. This paper proposes a joint adaptation of the MIMO physical layer and the 802.11e MAC layer through the formulation of a network utility maximization problem. The MIMO configuration at the physical layer and the contention window sizes for different access categories' traffic at the MAC layer are jointly optimized. Simulations are carried out in the ns-2 simulator to show the effectiveness of the proposed method.
\end{abstract}

\section{INTRODUCTION}

The IEEE 802.11-based wireless local area networks (WLANs) have been widely recognized as one of the most successful technologies for providing high-speed wireless data access for hotspot locations. The 802.11e standard [1] further introduced distributed quality-of-service (QoS) support with the Enhanced Distributed Channel Access (EDCA) function. EDCA is a contention-based access control scheme. The traffic are classified into four different access categories (ACs), where each $\mathrm{AC}$ has different priority for channel access based on its own minimum and maximum contention window $(\mathrm{CW})$ size, Arbitration Inter-Frame Space (AIFS), and transmission opportunity values. Among these parameters, the minimum contention window size has been shown to have a major impact on the network performance [2]. Various schemes have been proposed to adaptively tuning the $\mathrm{CW}$ value to improve the MAC efficiency [3], [4]. In this work, we will consider the adaptation of the $\mathrm{CW}$ at the 802.11e MAC layer, jointly with multi-antenna transmission schemes at the physical layer.

With the multiple-input-multiple-output (MIMO) technology at the physical layer, the recent $802.11 \mathrm{n}$ proposal [5] aims to significantly improve the physical link data rate up to 600 Mbps. Multiple antennas can achieve significant performance improvements over the conventional single-antenna systems. In a wireless fading channel with sufficiently rich scattering, two major performance gains can be realized in a wireless
MIMO system: the diversity gain and the spatial multiplexing gain [6]. The spatial diversity gain is realized when we transmit the same information symbols across different fading paths. The chance of all the signal paths experiencing deep fading simultaneously is greatly reduced. Thus, the transmission reliability is significantly enhanced with spatial diversity. On the other hand, if we transmit independent data symbols over each transmission antenna, the spatial multiplexing gain can be achieved, which significantly increases the spectral efficiency.

To quantify the MIMO gains, the multiplexing gain $r$ and diversity gain $d$ have been formally defined in [7] as:

$$
\lim _{\gamma \rightarrow \infty} \frac{R(\gamma)}{\log \gamma}=r, \text { and } \lim _{\gamma \rightarrow \infty} \frac{\log P_{e}(\gamma)}{\log \gamma}=-d,
$$

where $\gamma$ is the average signal-to-noise-ratio (SNR) of the wireless link, $R(\gamma)$ and $P_{e}(\gamma)$ are the link data rate and the average error probability, respectively.

For a point-to-point wireless link with $M_{T}$ transmit antennas and $M_{R}$ receive antennas, the maximal (full) diversity gain is $M_{T} M_{R}$. The maximal spatial multiplexing gain is $\min \left\{M_{T}, M_{R}\right\}$. However, the two gains cannot be fully realized at the same time. There is a fundamental tradeoff between these two performance gains. For each spatial multiplexing gain $r$, the best diversity gain $d^{*}(r)$ is the supremum of the diversity gain achieved over all schemes. In a Rayleighfading channel with long enough block lengths, the optimal multiplexing-diversity tradeoff $d^{*}(r)$ is given by the piecewiselinear function connecting the points $\left(r, d^{*}(r)\right)$ [7], [8]:

$$
d^{*}(r)=\left(M_{T}-r\right)\left(M_{R}-r\right),
$$

where $r=0,1, \ldots, \min \left\{M_{T}, M_{R}\right\}$.

In this paper, we assume that a family of carefully designed codes can achieve the above optimal tradeoff performance. The design of such codes under different antenna and channel conditions is an active research area and is beyond the scope of this paper.

The traditional network protocol design has been following the layered architecture under the TCP/IP five-layer reference model. Recent development has found that cross-layer design [9] is critical for performance optimization in a wireless communication paradigm due to the close coupling of the physical layer's characteristics with higher layers' performances. With the addition of the MIMO technique to the physical layer, active research has been going on in investigating the effects of 
MIMO on high-layer protocols, and searching for better crosslayer designs that are adaptive to the richer features provided by MIMO.

Some cross-layer designs focus on utilizing one of the two fundamental MIMO resources: either diversity or spatial multiplexing. For example, a MIMO-enabled MAC protocol based on CSMA/CA in an ad-hoc network was proposed in [10], where the MAC layer carefully schedules the transmission of different spatial streams by utilizing the spatial multiplexing capability of the MIMO system.

With better flexibility, we can enable the system to adaptively switch between these two MIMO working modes. Toledo and Wang [11] studied the performance of the transmission control protocol (TCP) over wireless MIMO channels. They concluded that at lower SNR regions, it is better to work under larger diversity gains which provide a more reliable wireless link. But at higher SNR regions, a coding scheme with higher multiplexing gain outperforms diversity schemes.

However, as shown in (2), apart from the two extreme working points where we obtain a maximal multiplexing gain $r$ while the diversity gain $d$ is zero (or vice versa), there are intermediate working modes where we can achieve part of the diversity gain and part of the multiplexing gain, simultaneously. Intuitively, adaptively tuning the MIMO system's operation mode along the optimal trade-off curve provided by (2) might provide useful system gains. In [12], Lee et al. studied the optimal tradeoff in a MIMO ad-hoc network by formulating the rate-reliability tradeoff problem as a network utility maximization (NUM) problem. However, only one class of traffic is considered, and the MAC uses a reservation based scheduling scheme, where the transmission time by each node has to be allocated in advance.

In this paper, we study the cross-layer design of a 802.11ebased WLAN with MIMO channels by jointly optimizing the $\mathrm{CW}$ at the MAC layer and the multiplexing-diversity tradeoff at the physical layer. Two $\mathrm{CW}$ adaptation schemes are proposed, and the problem is formulated as a NUM problem.

The contributions of our work are as follows:

1) Two system models for an 802.11e WLAN with different assumptions on the adaptation capability of the contention window sizes are proposed.

2) We propose two cross-layer schemes, namely $U$-MAC and $D-M A C$, which jointly select the MIMO coding scheme at the physical layer and the minimum $\mathrm{CW}$ size at the MAC layer to achieve the maximal network utility.

3) Simulation results by the ns- 2 simulator are presented to study the effectiveness of the proposed schemes and compared with the original 802.11e MAC.

The rest of the paper is organized as follows. In Section II, the system model and network saturation throughput analysis are presented. Section III proposes the NUM optimization framework for the two system models. Section IV presents the simulation results and performance comparisons. Conclusions and future work are given in Section V.

\section{SySTEM MODEL}

Consider a WLAN with an access point (AP). Let $\mathcal{N}$ denote the set of wireless stations. According to the EDCA specifications in the 802.11e standard, there can be up to four different classes of traffic with different QoS requirements. For simplicity, we study the case of two access categories (ACs) ( $A C_{1}$ and $A C_{2}$ ), with $A C_{2}$ being the higher priority traffic in the network. We use $\mathcal{N}_{1}$ and $\mathcal{N}_{2}$ to denote the set of stations in each AC. Each wireless station transmits one class of traffic, so the set of all stations $\mathcal{N}$ is $\mathcal{N}_{1} \cup \mathcal{N}_{2}$. We use $N_{1}, N_{2}$, and $N$ to denote the number of stations in each set. Extension to four ACs is fairly straightforward based on the optimization framework proposed below.

We study the performance of wireless stations competing for channel access and sending data to the AP. Each wireless station has $M_{T}$ transmit antennas, and the AP has $M_{R}$ receiving antennas. From Section I, we assume that each wireless link can utilize some multi-antenna coding scheme to achieve the optimal diversity-multiplexing tradeoff given by (2). However, this curve is non-differentiable which may cause problems for solving the NUM problem. We use the differentiable approximation as in [12]:

$$
d(r)=\left(M_{T}-r\right)\left(M_{R}-r\right), \quad 0 \leq r \leq \min \left\{M_{T}, M_{R}\right\} .
$$

If a wireless link from the wireless station $s \in N$ to the AP has signal-to-noise ratio $\gamma_{s}$, multiplexing gain $r_{s}$ and diversity gain $d_{s}$, then the link data rate $c_{s}\left(\gamma_{s}\right)$ (bps) and error probability $p_{s}^{e r r}\left(\gamma_{s}\right)$ can be approximated as [13]:

$$
c_{s}\left(\gamma_{s}\right)=k_{c} r_{s} \log \gamma_{s}, \quad \text { and } p_{s}^{e r r}\left(\gamma_{s}\right)=k_{p} \gamma_{s}^{-d\left(r_{s}\right)}
$$

where $k_{c}$ and $k_{p}$ are positive constants for different coding schemes. The log function uses base 2 . The multiplexing gain $r_{s}$ and diversity gain $d_{s}$ conform to the optimal tradeoff designated by (3). Then, the link transmission reliability $y_{s}$ is:

$$
y_{s}=1-k_{p} \gamma_{s}^{-d\left(r_{s}\right)}, \quad s \in \mathcal{N} .
$$

With $c_{s}$ being the data rate of wireless station $s$, the achievable throughput of each station is usually significantly lower than $c_{s}$ due to the CSMA/CA random contention and the protocol overhead [2]. To formulate the NUM problem, we need an analytical description of each station's throughput in relation to $c_{s}$ and other network parameters, which is a difficult task due to the CSMA/CA random access scheme. However, the saturation throughput is more tractable and has been successfully derived from various mathematical models.

The saturation throughput of an 802.11 network is a good indicator of network performance because it is the sustainable throughput under heavy traffic load, and is also very close to the network maximum capacity. In this work, we base our analysis on the saturation performance of the network. There are two causes of packet loss in an 802.11 network. One is packet collision where two nodes transmit simultaneously. The other one is channel error, where a packet is received free of collisions, but is corrupted due to low SNR. 
We denote the saturation throughput under an ideal channel (no packet errors) with $x_{s}$. Then, $x_{s} y_{s}$ can approximately represent the effective throughput after further discarding the channel corrupted packets. This is not the most accurate model for error-prone WLANs, because the channel error also has an impact on the backoff process. However, this approximation separates the effects of collision and channel error on the throughput, and greatly facilitates the problem formulation.

The throughput $x_{s}$ is primarily a function of the physical layer parameters, such as the network basic and data rates, and the MAC layer parameters such as contention window sizes, the DIFS/SIFS/AIFS values, and frame header overhead. Of all the elements listed above, we focus on studying the effects of the data rate $c_{s}$ and the minimum contention window size $W_{s}$ in this paper. The data rate with a MIMO channel is given by (4), and is a function of the SNR $\gamma_{s}$ and each station's MIMO operation mode $r_{s}$. We consider two cases of the $W_{s}$ assignment. One is that there is a uniform $\mathrm{CW}$ value for each $\mathrm{AC}$, where all stations belonging to the same $\mathrm{AC}$ use the same $\mathrm{CW}$ value. This is the normal behavior in 802.11e. We call this MAC as the Uniform-CW MAC (U-MAC). The other one is that we allow each wireless station to freely choose its own $\mathrm{CW}$ value without conforming to the same $\mathrm{CW}$ for its $\mathrm{AC}$. We call this MAC as the Differentiated-CW MAC (D-MAC).

Time is partitioned into virtual time slots where each slot can be either a backoff time slot, a collision-free transmission slot, or a collided transmission slot. The probability for a station $s$ to transmit in a virtual slot is [2]:

$$
\tau_{s}=\frac{2}{W_{s}+1}, \quad s \in \mathcal{N},
$$

where $W_{s}$ is stations $s$ 's minimum $\mathrm{CW}$ size. The probability that one transmitted packet by station $s$ will collide is the probability that any of the other stations also transmits:

$$
q_{s}=1-\prod_{k \in N, k \neq s}\left(1-\tau_{k}\right), \quad s \in \mathcal{N} .
$$

With the above results for $\tau_{s}$ and $q_{s}$, we analyze $x_{s}$ for the two MAC schemes separately below.

\section{A. U-MAC: With Uniform CW for Stations in Each $A C$}

For the $U-M A C, \tau_{s}$ and $q_{s}$ in (6) and (7) are reduced to the same values for each AC. To calculate $x_{s}$, we extend the mean value based saturation throughput analysis in [14]. The main difference here is that the wireless stations may use different data rates other than the uniform data rate assumed in [14]. The average length of one virtual time slot $T_{a}$ for each AC can be approximated as:

$$
\begin{aligned}
T_{a}= & \sum_{n \in \mathcal{N}_{a}} \frac{\tau_{n}}{\sum_{k \in \mathcal{N}_{a}} \tau_{k}} \frac{L}{c_{n}}+T_{h d r}+T_{S I F S}+T_{A C K} \\
& +T_{A I F S}+\frac{\bar{W}_{a}}{N_{a}+1} T_{\text {slot }}, \quad a=1,2,
\end{aligned}
$$

where $T_{h d r}, T_{A C K}, T_{\text {slot }}, T_{S I F S}$ and $T_{A I F S}$ are the length of the frame header, the ACK frame, the backoff time slot length, the SIFS and AIFS length, respectively. $\bar{W}_{a}$ (for $a=$
$1,2)$ represents the minimum contention window size of $A C_{a}$. The same AIFS is used for each AC. An identical payload length $L$ is assumed for the network. $L / c_{n}$ is the time to transmit the frame payload of station $n$. The average payload transmission time is the weighted sum of all $A C_{a}$ stations, using each station's transmission probability as the weight.

By omitting the detailed steps from [14], we can calculate the rate of success for $A C_{a}$, which is the average number of collision-free transmissions by $A C_{a}$ per second, as:

$$
v_{a}=N_{a} \tau_{a}\left(1-q_{a}\right) / \sum_{k=1}^{2}\left(1-q_{k} / 2\right) N_{k} \tau_{k} T_{k}, \quad a=1,2 .
$$

The throughput for station $s$ in each AC can be calculated as:

$$
x_{s}=v_{a} L \frac{c_{s}\left(\gamma_{s}\right)}{\sum_{i \in \mathcal{N}_{a}} c_{i}\left(\gamma_{i}\right)}, \quad \forall s \in \mathcal{N}_{a}, \quad a=1,2,
$$

The above equation shows that the individual throughput of each station $s$ in $A C_{a}$ is the weighted portion of $v_{a} L$ in proportion to its data rate $c_{s}$.

\section{B. D-MAC: With Different CW for Each Station}

For the $D-M A C$, we allow each station $s$ to choose its own $W_{s}$ value individually based on its link state and class of traffic. In this case, we have an 802.11e WLAN with multiple data rates, and multiple $\mathrm{CW}$ values. To model this system accurately is a challenging task. Even for the basic 802.11e MAC, complicated multi-dimensional Markov chain and queueing theories have to be used, which result in complex systems of non-linear equations to solve for the throughput $x_{s}$ [15]. Although these models give accurate results, they usually require some stringent assumptions, and none can handle a versatile system where each wireless station has a distinct data rate and a distinct $\mathrm{CW}$.

However, we may not need a highly accurate throughput model for the NUM framework to work properly. Other factors, such as the convergence of the optimization problem and computation efficiency, are important for a NUM problem. It is preferable to obtain a closed form expression of $x_{s}$ instead of a system of nonlinear equations on $x_{s}$. With this goal, we propose that the throughput of station $s$ be modeled as:

$$
x_{s}=\Gamma \tau_{s}\left(1-q_{s}\right) \frac{L}{T_{a}}, \quad s \in \mathcal{N}_{a}, \quad a=1,2,
$$

where $T_{a}(a=1,2)$ is the average virtual slot length as defined in (8), but with $\bar{W}_{a}$ in (8) representing the average CW of all stations in $A C_{a} . \Gamma$ is a positive scaling factor. Here, we assume that the two ACs have the same scaling factor. In Section III, we can see that the exact value of $\Gamma$ need not be known for solving the NUM problem.

The above equations state that the throughput of wireless station $s$ is in proportion to its transmission probability $\tau_{s}$, collision-free probability $\left(1-q_{s}\right)$, and the average transmission rate of one payload by each $A C_{a}\left(L / T_{a}\right)$. This is reasonable assumption as a station's probability of transmission and collision are the major factors influencing its throughput. The linear proportional relationship is a bold assumption. From our later 
simulation results, we can verify that our NUM formulation based on this simple assumption can efficiently achieve the desired traffic differentiation and utility optimization effects.

\section{Network Utility Formulation}

The utility function for each source node in a NUM framework is usually chosen such that it reflects the satisfaction attained by the node with the network services it receives, or the revenue that the network operator would be able to accrue by providing a certain level of QoS service to the end users. A commonly used family of utility functions parameterized by $\alpha \geq 0$ is proposed in [16]:

$$
U(x)= \begin{cases}(1-\alpha)^{-1} x^{(1-\alpha)}, & \text { if } \alpha \neq 1, \\ \log x, & \text { if } \alpha=1 .\end{cases}
$$

The parameter $\alpha$ affects the level of fairness achieved in the network. For example, if $x$ is each source node's throughput, $\alpha=0$ leads to network throughput maximization. Proportional, harmonic mean and max-min fairness are achieved when $\alpha=1, \alpha=2$, and $\alpha \rightarrow \infty$, respectively.

In our NUM framework, we choose the utility function for each wireless station $s$ with this form:

$$
U_{s}\left(x_{s}, y_{s}\right)=(1-\alpha)^{-1}\left(x_{s} y_{s}\right)^{1-\alpha}, \quad \alpha>1,
$$

where $x_{s} y_{s}$ is the effective throughput of node $s$ after accounting for both collisions and channel errors.

The network utility function is defined as:

$$
U(\mathbf{x}, \mathbf{y})=\sum_{i \in \mathcal{N}_{1}} \beta U_{1}\left(x_{i}, y_{i}\right)+\sum_{j \in \mathcal{N}_{2}}(1-\beta) U_{2}\left(x_{j}, y_{j}\right) . \alpha>1,
$$

where $\beta$ is an adjustable parameter between 0 and 0.5 to tune the weight of $A C_{1}$ and $A C_{2}$ on the network utility. It can be proved that $U(\mathbf{x}, \mathbf{y})$ is a concave function of $\mathbf{x}, \mathbf{y}$.

\section{NUM FRAMEWORK FOR CROSS-LAYER DESIGN}

With the system model and definition of the network utility, we propose our NUM formulation for cross-layer design of a MIMO-enabled 802.11e WLAN, where each station's MIMO multiplexing gain at the physical layer and the contention window sizes at the MAC layer are jointly optimized.

\section{A. $U-M A C$}

With $A C_{a}$ 's stations using a contention window size of $C W_{a}$, the NUM problem can be formulated as:

$$
\begin{aligned}
& \max _{\mathbf{x}, \mathbf{y}} \sum_{i \in \mathcal{N}_{1}} \beta U_{1}\left(x_{i}, y_{i}\right)+\sum_{j \in \mathcal{N}_{2}}(1-\beta) U_{2}\left(x_{j}, y_{j}\right) \\
& \text { s.t. } \quad 0 \leq x_{s} \leq v_{a} L \frac{c_{s}\left(\gamma_{s}\right)}{\sum_{n \in \mathcal{N}_{a}} c_{n}\left(\gamma_{n}\right)}, \forall s \in \mathcal{N}_{a}, a=1,2 \\
& \check{y}_{a} \leq y_{s} \leq 1-k_{p} \gamma_{s}^{-\left(M_{T}-r_{s}\right)\left(M_{R}-r_{s}\right)} \text {, } \\
& \forall s \in \mathcal{N}_{a}, a=1,2 \\
& 0 \leq r_{s} \leq \min \left\{M_{T}, M_{R}\right\}, \quad \forall s \in \mathcal{N} \\
& \check{\bar{W}}_{a} \leq \bar{W}_{a} \leq \overline{\bar{W}}_{a}, \quad a=1,2,
\end{aligned}
$$

where $v_{a}$ is given by (9), and are functions of $\bar{W}_{a}, r_{s}$ and other system paramters. The $\check{y}_{a}, \check{W}_{a}$ and $\bar{W}_{a}$ are constant bounds on the variables, and should be selected within reasonable ranges. The above problem is a NUM problem with variables $\mathbf{x}, \mathbf{y}, \mathbf{r}$, and $\bar{W}_{a}$. We relaxed the equality of $x$ (10) to inequality in the above formulation, because a nonlinear inequality constraint is usually easier to handle than an equality constraint for nonlinear optimizations. Also, because the utility objective function is strictly increasing in $x$, we would expect that this upper bound constraint on $x$ will always be tight. Although the objective function in (15) is concave, the constraint sets make this problem non-convex and challenging to solve. However, our tests with Matlab's nonlinear optimization toolbox, using the sequential quadratic programming (SQP) method [17], show that this problem formulation, under reasonable ranges of network parameters and with varying initial variable values, has no difficulty in converging to a unique solution. The number of iterations needed is in the range of 20 to 200 .

\section{B. D-MAC}

With each station $s$ choosing its contention window size $W_{s}$ individually, the NUM problem can be formulated as:

$$
\begin{aligned}
& \max _{\mathbf{x}, \mathbf{y}} \sum_{i \in \mathcal{N}_{1}} \beta U_{1}\left(x_{i}, y_{i}\right)+\sum_{j \in \mathcal{N}_{2}}(1-\beta) U_{2}\left(x_{j}, y_{j}\right) \\
& \text { s.t. } \quad 0 \leq x_{s} \leq \Gamma \tau_{s}\left(1-q_{s}\right) \frac{L}{T_{a}}, \quad \forall s \in \mathcal{N}_{a}, a=1,2 \\
& \check{y}_{a} \leq y_{s} \leq 1-k_{p} \gamma_{s}^{-\left(M_{T}-r_{s}\right)\left(M_{R}-r_{s}\right)} \text {, } \\
& \forall s \in \mathcal{N}_{a}, a=1,2 \\
& 0 \leq r_{s} \leq \min \left\{M_{T}, M_{R}\right\}, \quad \forall s \in \mathcal{N} \text {, } \\
& \check{W}_{s} \leq W_{s} \leq \hat{W}_{s}, \quad \forall s \in \mathcal{N} \text {, }
\end{aligned}
$$

where $\hat{W}_{s}$ and $\check{W}_{s}$ are the lower and upper bounds of each station's $\mathrm{CW}$ value. The variables $\tau_{s}, q_{s}$, and $T_{a}$ are given in (6)-(8). $x_{s}$ 's equality constraint has also been relaxed to inequality as explained before. We can also observe that the effect of the constant parameter $\Gamma$ is that it ends up being a scaling factor $\Gamma^{1-\alpha}$ in the utility function, and has no effect on our optimal solution. The above problem is a NUM problem with variables $\mathbf{x}, \mathbf{y}, \mathbf{r}$, and $W_{s}$. It can also be solved using standard algorithms for constrained nonlinear optimization problems as discussed in Section III-A.

With the above $U-M A C$ and $D-M A C$ formulation, the AP in an 802.11e WLAN can collect each wireless station's link SNR, select a $\beta$ with appropriate network revenue models, and solve either (15) or (16) to obtain the MIMO multiplexing gain $r_{s}$ (rounded to the nearest integer) and minimum contention window size $W_{s}$ for each station. The resulting $r_{s}$ and $W_{s}$ values can be sent out in the EDCA parameter set elements of the 802.11e beacon frames for each station to adjust its physical and MAC layer operation parameters.

\section{Simulation Results}

We carry out network simulations in the ns-2 [18] simulator to study the effectiveness of the cross-layer optimization framework proposed in the last section. The simulation parameters are shown in Table I. 
TABLE I

Simulation PARAMETERS

\begin{tabular}{|l|c|}
\hline \hline Basic Rate & $10 \mathrm{Mbps}$ \\
\hline Maximum Data Rate & $80 \mathrm{Mbps}$ \\
\hline DSSS PLCP Preamble and Header & $192 \mathrm{bits}$ \\
\hline MAC Header & $192 \mathrm{bits}$ \\
\hline FCS (Frame Check Sequence) & $32 \mathrm{bits}$ \\
\hline ACK Frame Size (include Headers) & $304 \mathrm{bits}$ \\
\hline Data Packet Size & $1000 \mathrm{bytes}$ \\
\hline Time Slot & $9 \mu \mathrm{s}$ \\
\hline SIFS & $10 \mu \mathrm{s}$ \\
\hline Maximum Number of Retransmissions & 5 \\
\hline
\end{tabular}

We test a WLAN with 10 stations. Five of them have $A C_{1}$ traffic, and five of them have $A C_{2}$ traffic. To effectively study proposed scheme's traffic differentiation effects, we use the same SNR values for each pair of $A C_{1}$ and $A C_{2}$ stations. That is $\gamma_{i}=\gamma_{i+5}(i=1, \ldots, 5)$. For $\gamma_{i},(i=1, \ldots, 5)$, they are randomly chosen from gaussian distributed random numbers with a mean value of $10 \mathrm{~dB}$, and a variance of 5 . This resembles a WLAN with wireless stations randomly located with similar distances from the AP. For $U-M A C$ and $D-M A C$, the following parameters are used: $k_{c}=20 \mathrm{MHz}, k_{p}=0.15$, $\check{W}_{s}=\check{W}_{a}=7, \hat{W}_{s}=\hat{W}_{a}=1023, \alpha=1.1, \check{y}_{1}=0.7$, $\check{y}_{2}=0.85$, and $\Gamma=1$. With a maximum of 5 retransmissions, the maximum $\mathrm{CW}$ is set to be $2^{5}$ times the minimum $\mathrm{CW}$. The same AIFS of $28 \mu \mathrm{s}$ is used for both ACs. The resulting MIMO multiplexing gain $r$ and contention window size $W$ from the solution of (15) or (16) are used in the ns-2 simulator to assign the proper physical and MAC operating parameters. We vary the utility parameter $\beta$ from 0.1 to 0.5 to study the effectiveness of the schemes' traffic differentiation effect. Smaller $\beta$ improves the higher priority $A C_{2}$ 's performance, but at a price of decreasing performance of $A C_{1}$. A desired tradeoff may be obtained by assigning the appropriate $\beta$ by the network administrator.

We compare the proposed adaptive cross-layer schemes $U$ $M A C$ and $D-M A C$ with the original 802.11e MAC. For the original 802.11e MAC, the $W_{1}$ and $W_{2}$ are chosen as 7 and 31 respectively, according to the standard specifications. In order to have meaningful performance comparison, we also use MIMO links with the 802.11e MAC. But each station's multiplexing gain $r_{s}$ is chosen without cross-layer optimization as done in the $U-M A C$ and $D-M A C$ schemes. Its $r_{s}$ is chosen by (5) such that wireless station $s$ would achieve a link reliability of 0.9 with its channel SNR $\gamma_{s}$.

Figures 1 and 2 show the aggregated throughput of each $\mathrm{AC}$ and the throughput's standard deviation. The standard deviation value shows the fluctuation of each AC's throughput and is a good representation of the quality of service provided by the network. Because of the static nature of the $802.11 \mathrm{e}$ MAC scheme, its performance values do not change with $\beta$, and are drawn as a horizontal line in each figure to be used as a performance comparison baseline. The medium access delay and the delay's standard deviation are shown in Figures 3 and 4 respectively. As we would expect, an increase in $\beta$ decreases the priority differentiation between the two ACs.

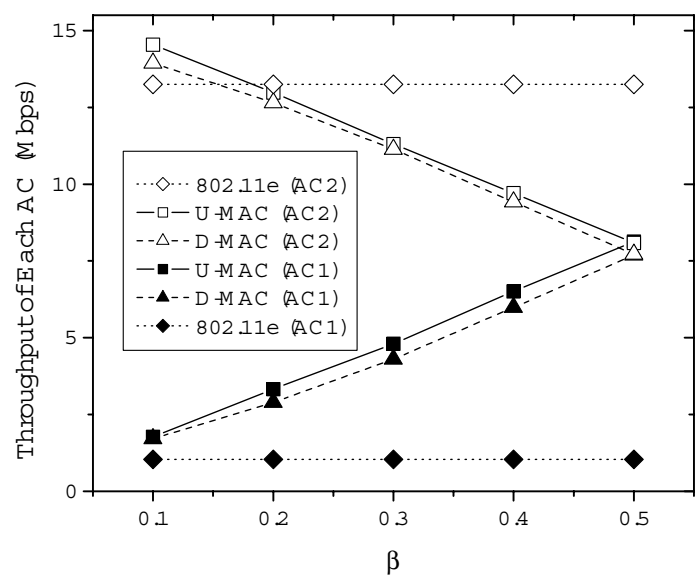

Fig. 1. Average throughput between U-MAC, D-MAC, and 802.11e.

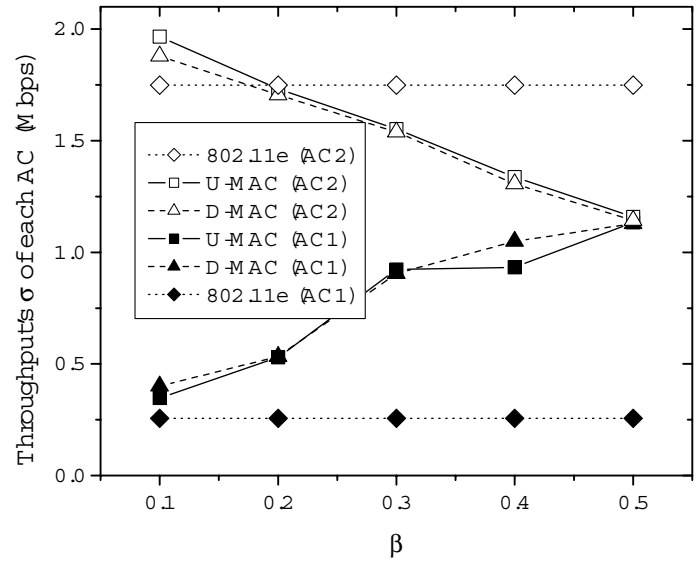

Fig. 2. Performance of average standard deviation of throughput.

When $\beta$ reaches $0.5, A C_{1}$ and $A C_{2}$ reach equal priority and have comparable performances.

The network utility is shown in Figure 5. Although the 802.11e MAC does not consider $\beta$, its $x_{s}, y_{s}$ values are also substituted into (14) to calculate the network utility under different $\beta$. Figure 5 shows that the $U-M A C$ and $D-M A C$ maintain a relatively high network utility, while the $802.11 \mathrm{e}$ is not adaptive to different $\beta$ adjustments and its network utility decreases sharply when the utility function demands less traffic differentiation by increasing the $\beta$ value.

From all the simulation results, we can observe that the adaptive $U-M A C$ and $D-M A C$ schemes have good flexibility in adapting to network's different assignment of weights to the two ACs. They also consistently outperform the original 802.11e MAC in every performance measure because they jointly adapt the $\mathrm{CW}$ values and the MIMO coding schemes. These results verify that the several simplifying assumptions that we made to formulate the computationally efficient NUM problem in Section II are reasonable and sound.

We can also observe that the $U-M A C$ slightly outperforms the $D-M A C$, even though $D-M A C$ allows the extra flexibility of letting each station have its own $W_{s}$ parameter. The cause for this may be from the coarse approximation we made in 


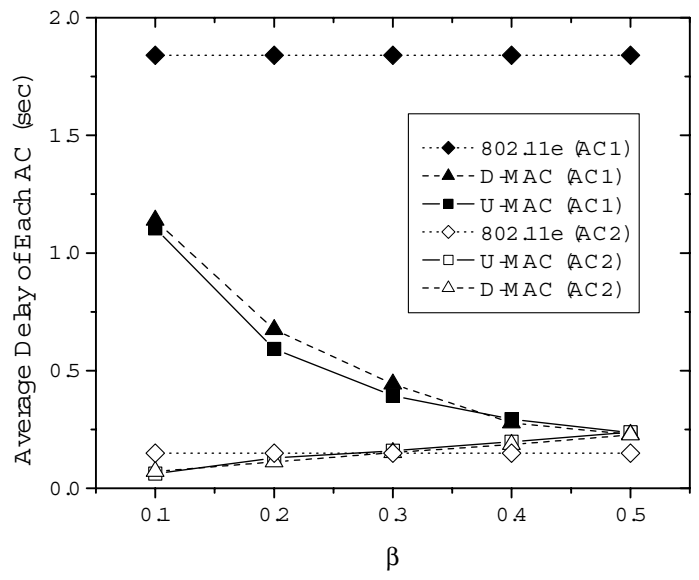

Fig. 3. Average delay between U-MAC, D-MAC, and 802.11e.

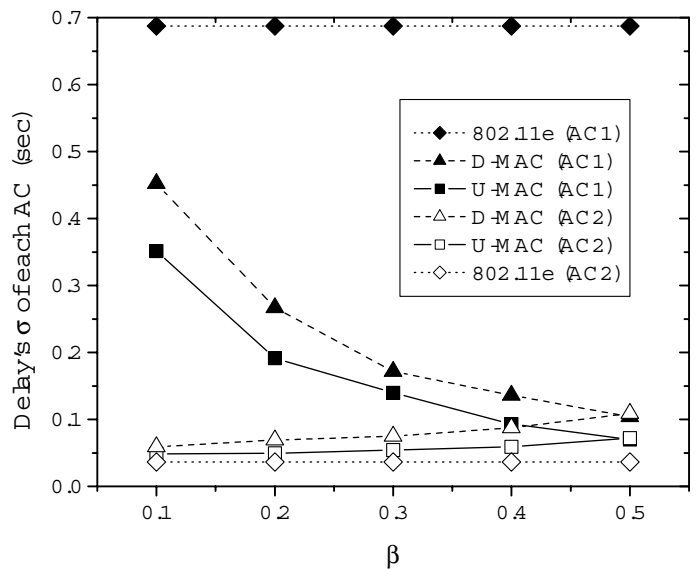

Fig. 4. Performance of average standard deviation of delay.

$D$-MAC's throughput model, while the $U-M A C$ model is more accurate. However, an insight that we can gain from these simulation experiments is that restricting each $\mathrm{AC}$ to have uniform $\mathrm{CW}$ values may be sufficient enough to provide good performance gain in the MIMO-enabled cross layer design framework. We may be able to improve $D-M A C$ 's performance by further refining its throughput model.

\section{CONCLUSIONS}

This paper proposed a MAC/PHY cross-layer design for network utility maximization in an 802.11e WLAN with multiple classes of traffic. The minimum contention size at the MAC layer is jointly optimized with the multiplexing-diversity gain tradeoff at the physical layer with MIMO antennas. The utilitybased cross-layer design is shown to be flexible in adjusting the system performance in regard to QoS tradeoff for different access categories of traffic. However, the current schemes work in a centralized manner. Extending the framework to work in a distributed manner and possibly in a multi-hop mesh network environment are part of our future work.

\section{ACKNOWLEDGMENT}

This work is supported by the Natural Sciences and Engineering Research Council of Canada.

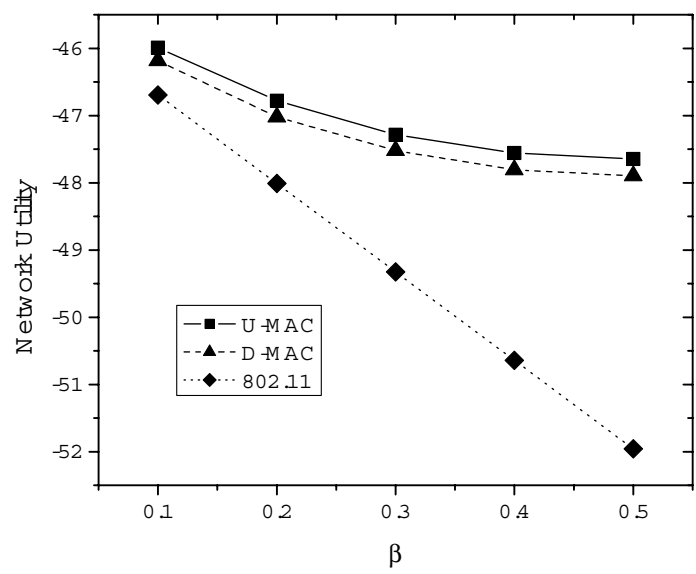

Fig. 5. Network utility of U-MAC, D-MAC, and 802.11 versus $\beta$.

\section{REFERENCES}

[1] IEEE 802.11e WG, "Wireless LAN MAC and PHY specifications amendment 8: MAC quality of service enhancements," Sept. 2005.

[2] G. Bianchi, "Performance analysis of the IEEE 802.11 distributed coordination function," IEEE J. Select. Areas Commun., vol. 18, no. 3, pp. 535-547, Mar. 2000.

[3] F. Cali, M. Conti, and E. Gregori, "Dynamic tuning of the IEEE 802.11 protocol to achieve a theoretical throughput limit," IEEE/ACM Trans. Networking, vol. 8, no. 6, pp. 785-799, Dec. 2000.

[4] A. L. Toledo, T. Vercauteren, and X. Wang, "Adaptive optimization of IEEE 802.11 DCF based on bayesian estimation of the number of competing terminals," IEEE Trans. Mobile Comput., vol. 5, no. 9, pp. 1283-1296, Sept. 2006.

[5] IEEE 802.11n WG, "IEEE 802.11n draft 2.0," Jan. 2007.

[6] D. Tse and P. Viswanath, Fundamentals of Wireless Communication. Cambridge University Press, 2005.

[7] L. Zheng and D. N. C. Tse, "Diversity and multiplexing: A fundamental tradeoff in multiple-antenna channels," IEEE Trans. Inform. Theory, vol. 49, no. 5, pp. 1073-1096, May 2003.

[8] A. Medles and D. T. M. Slock, "Achieving the optimal diversity-versusmultiplexing tradeoff for MIMO flat channels with QAM spacectime spreading and DFE equalization," IEEE Trans. Inform. Theory, vol. 52, no. 12, pp. 5312-5323, Dec. 2006.

[9] X. Lin, N. Shroff, and R. Srikant, "A tutorial on cross-layer optimization in wireless networks," IEEE J. Select. Areas Commun., vol. 24, no. 8, pp. 1452-1463, Aug. 2006.

[10] K. Sundaresan, R. Sivakumar, M. A. Ingram, and T. Chang, "Medium access control in ad hoc networks with MIMO links: optimization considerations and algorithms," IEEE Trans. Mobile Comput., vol. 3, no. 4, pp. 350-365, Oct.-Dec. 2004.

[11] A. L. Toledo and X. Wang, "TCP performance over wireless MIMO channels with ARQ and packet combining," IEEE Trans. Mobile Comput., vol. 5, no. 3, pp. 208-223, Mar. 2006.

[12] J. Lee, M. Chiang, and A. R. Calderbank, "Price-based distributed algorithms for rate-reliability tradeoff in network utility maximization," IEEE J. Select. Areas Commun., vol. 24, no. 5, pp. 962-976, May 2006.

[13] D. Gesbert, M. Shafi, D. Shiu, P. J. Smith, and A. Naguib, "From theory to practice: an overview of MIMO space-time coded wireless systems," IEEE J. Select. Areas Commun., vol. 21, no. 3, pp. 281-302, Apr. 2003.

[14] Y. Lin and V. Wong, "Saturation throughput of IEEE 802.11e EDCA based on mean value analysis," in Proc. of IEEE WCNC, Las Vegas, Nevada, Apr. 2006.

[15] Z. Kong, D. Tsang, and B. Bensaou, "Performance analysis of IEEE 802.11 e contention-based channel access," IEEE J. Select. Areas Commun., vol. 22, no. 10, pp. 2095-2106, Dec. 2004.

[16] J. Mo and J. Walrand, "Fair end-to-end window-based congestion control," IEEE/ACM Trans. Networking, vol. 8, no. 5, Oct. 2000.

[17] A. R. Conn, N. I. M. Gould, and P. L. Toint, Trust-region Methods. Society for Industrial Mathematics, 2000.

[18] Ns-2 simulator. [Online]. Available: http://www.isi.edu/nsnam/ns/ 